\title{
Global conservative solutions of the Camassa-Holm equation
}

\author{
Alberto Bressan \\ Deptartment of Mathematics, Pennsylvania State University, University Park 16802, U.S.A. \\ e-mail: bressan@math.psu.edu \\ and \\ Adrian Constantin \\ Department of Mathematics, Lund University, 22100 Lund, Sweden \\ Trinity College, Department of Mathematics, Dublin 2, Ireland \\ e-mail: adrian.constantin@math.lu.se
}

\begin{abstract}
This paper develops a new approach in the analysis of the Camassa-Holm equation. By introducing a new set of independent and dependent variables, the equation is transformed into a semilinear system, whose solutions are obtained as fixed points of a contractive transformation. These new variables resolve all singularities due to possible wave breaking. Returning to the original variables, we obtain a semigroup of global solutions, depending continuously on the initial data. Our solutions are conservative, in the sense that the total energy equals a constant, for almost every time.
\end{abstract}

\section{0 - Introduction}

The nonlinear partial differential equation

$$
u_{t}-u_{t x x}+3 u u_{x}=2 u_{x} u_{x x}+u u_{x x x}, \quad t>0, x \in \mathbb{R},
$$

was derived by Camassa and Holm $[\mathrm{CH}]$ as a model for the propagation of shallow water waves, with $u(t, x)$ representing the water's free surface over a flat bed (see also the alternative derivation in $[\mathrm{J}])$. The Camassa-Holm equation was actually obtained much earlier as an abstract bi-Hamiltonian partial differential equation with infinitely many conservation laws by Fokas and Fuchssteiner [FF] (see $[\mathrm{L}]$ ). Nevertheles, Camassa and Holm put forward its derivation as a model for shallow water waves and discovered that it is formally integrable (in the sense that there is an associated Lax pair) and that its solitary waves are solitons (i.e. the solitary waves retain their shape and speed after the nonlinear interaction with waves of the same type), features that prompted an ever increasing interest in the study of this equation. For a large class of initial data the Camassa-Holm equation is an integrable infinite dimensional Hamiltonian system. That is, by means of a Lax pair, it is possible to associate to each solution with initial data within this class some scattering data that evolve in time linearly at constant speed and from which the solution can be reconstructed in an explicit way (see [BSS2, CM1, C2]). In contrast to the Korteweg-de Vries equation, which is also an integrable model for shallow water waves, the Camassa-Holm equation possesses not only solutions that are global in time but models also wave breaking. Indeed, while some initial 
data develop into waves that exist indefinitely in time [CE1], others lead to wave breaking: the solution remains bounded but its slope becomes unbounded in finite time [CE2]. Moreover, wave breaking is the only way in which singularities can arise in a classical solution [C1]. This raises the natural question of the behaviour of the solutions after wave breaking. In [XZ1, XZ2] global weak solutions are obtained as weak limits of viscous regularizations of the Camassa-Holm equation. The notion of weak solution used in [XZ1, XZ2] allows for wave breaking so that a notion of solution independent of wave breaking or not is advanced. Nevertheless, due to lack of uniqueness (within the class of such global weak solutions, uniqueness is obtained only under an a priori assumption cf. [XZ2], and the fulfillment of this assumption can presently be guaranteed only for initial data that actually do not develop into breaking waves - see [CM2, W]), these investigations do not shed light on the wave breaking process or on what happens after breaking. Therefore, to gain insight into wave breaking, the most natural approach is to investigate it on some particular cases. The solitary waves of the Camassa-Holm equation are peaked waves: for each $c \in \mathbb{R}$, there is a solitary wave $\varphi_{c}$ (unique up to translations) propagating at speed $c$, given by the explicit formula

$$
\varphi_{c}(x-c t)=c e^{-|x-c t|}, \quad t>0, x \in \mathbb{R},
$$

cf. $[\mathrm{CH}]$. These solitary waves are stable solitons $[\mathrm{BSS} 1, \mathrm{CS}]$. Notice that the solitary waves are not classical solutions due to the fact that they have a peak at their crest (feature which explains why they are called "peakons"). They have to be understood [CM2] as weak solutions of the Camassa-Holm equation, for which purpose it is suitable to recast the equation in the nonlocal conservation law form

$$
u_{t}+u u_{x}+\partial_{x}\left(1-\partial_{x}^{2}\right)^{-1}\left(u^{2}+\frac{1}{2} u_{x}^{2}\right)=0 .
$$

The peakon-antipeakon interaction, corresponding to the evolution in time from an initial profile of the form $u(x, 0)=\varphi_{c}\left(x+x_{0}\right)-\varphi_{c}\left(x-x_{0}\right)$ with $c, x_{0}>0$ leads to wave breaking: $u_{x}(0, t) \rightarrow-\infty$ in finite time $T\left(c, x_{0}\right)>0$ cf. [CE1, W]. Two scenarios can be put forward. The first suggests total annihilation at $t=T\left(c, x_{0}\right)$ in the sense that $u(x, t)=0$ for all $t>T\left(c, x_{0}\right)$ (see [W]), whereas the second scenario proposes a "switching" phenomenon: the waves pass through each other, each continuing unscathed as a solitary wave (see $[\mathrm{BSS} 3, \mathrm{M}]$ ). The first scenario would correspond to loss of energy, whereas in the second the energy

$$
\int_{\mathbb{R}}\left(u^{2}+u_{x}^{2}\right) d x
$$

is preserved. Conservation of energy up to breaking time is of fundamental importance as the Camassa-Holm equation satisfies the Least Action Principle [C1].

The aim of this paper is to develop a new approach to the Camassa-Holm equation. In connection with smooth solutions, we first introduce a new set of independent and dependent variables. In these new variables, the evolution equation becomes semilinear. A local solution can be obtained as the fixed point of a contractive transformation. Remarkably, the new variables allow us to resolve all singularities. Indeed, solutions of the equivalent semilinear system remain smooth and can be globally extended in time, even after wave breaking. Going back to the original variables $u(t, x)$, we thus recover a semigroup of global solutions to the Camassa-Holm equation, continuously depending on the initial data. The solutions that we obtain are conservative, in the sense that their total energy, measured by the $H^{1}$ norm, is a.e. with respect to time equal to a constant. By a suitable modification in the dynamics of our semilinear system, it appears that one can also recover a continuous semigroup of dissipative solutions. These will be analyzed in a forthcoming paper. 


\section{1 - The basic equations}

We consider the following form of the Camassa-Holm equation

$$
u_{t}+\left(u^{2} / 2\right)_{x}+P_{x}=0,
$$

where the source term $P$ is defined as a convolution:

$$
P \doteq \frac{1}{2} e^{-|x|} *\left(u^{2}+\frac{u_{x}^{2}}{2}\right)
$$

As initial data, we take

$$
u(0, x)=\bar{u}(x), \quad x \in \mathbb{R},
$$

To make sense of the source term $P$, at each time $t$ we require that the function $u$ be in the space $H^{1}(\mathbb{R})$ of absolutely continuous functions $u \in \mathbf{L}^{2}(\mathbb{R})$ with derivative $u_{x} \in \mathbf{L}^{2}(\mathbb{R})$, endowed with the norm

$$
\|u\|_{H^{1}}=\left(\int_{\mathbb{R}}\left[u^{2}(x)+u_{x}^{2}(x)\right] d x\right)^{1 / 2} .
$$

For $u \in H^{1}(\mathbb{R})$, Young's inequality ensures that

$$
P=\left(1-\partial_{x}^{2}\right)^{-1}\left(u^{2}+\frac{1}{2} u_{x}^{2}\right) \in H^{1}(\mathbb{R}) .
$$

Definition 1. By a solution of the Cauchy problem (1.1)-(1.2) on $\left[t_{1}, t_{2}\right]$ we mean a Hölder continuous function $u=u(t, x)$ defined on $\left[t_{1}, t_{2}\right] \times \mathbb{R}$ with the following properties. At each fixed $t$ we have $u(t, \cdot) \in H^{1}(\mathbb{R})$. Moreover, the map $t \mapsto u(t, \cdot)$ is Lipschitz continuous from $\left[t_{1}, t_{2}\right]$ into $\mathbf{L}^{2}(\mathbb{R})$, satisfying the initial condition (1.2) together with

$$
\frac{d}{d t} u=-u u_{x}-P_{x}
$$

for a.e. $t$. Here (1.3) is understood as an equality between functions in $\mathbf{L}^{2}(\mathbb{R})$.

For smooth solutions, differentiating (1.1) w.r.t. $x$ one obtains

$$
u_{x t}+u u_{x x}+u_{x}^{2}-\left(u^{2}+\frac{u_{x}^{2}}{2}\right)+P=0 .
$$

Multiplying (1.1) by $u$ and (1.4) by $u_{x}$, we obtain the two conservation laws with source term

$$
\begin{gathered}
\left(\frac{u^{2}}{2}\right)_{t}+\left(\frac{u^{3}}{3}+u P\right)_{x}=u_{x} P, \\
\left(\frac{u_{x}^{2}}{2}\right)_{t}+\left(\frac{u u_{x}^{2}}{2}-\frac{u^{3}}{3}\right)_{x}=-u_{x} P,
\end{gathered}
$$

valid for smooth solutions. In particular, for regular solutions the total energy

$$
E(t) \doteq \int_{\mathbb{R}}\left(u^{2}(t, x)+u_{x}^{2}(t, x)\right) d x
$$


is constant in time, as one can see by first adding up (1.5) and (1.6) and subsequently integrating with respect to the $x$-variable. Since $P, P_{x}$ are both defined as convolutions, from the above bound on the total energy we immediately derive

$$
\begin{aligned}
& \|P(t)\|_{\mathbf{L}^{\infty}},\left\|P_{x}(t)\right\|_{\mathbf{L}^{\infty}} \leq\left\|\frac{1}{2} e^{-|x|}\right\|_{\mathbf{L}^{\infty}} \cdot\left\|u^{2}+\frac{u_{x}^{2}}{2}\right\|_{\mathbf{L}^{1}} \leq \frac{1}{2} E(0) . \\
& \|P(t)\|_{\mathbf{L}^{2}}, \quad\left\|P_{x}(t)\right\|_{\mathbf{L}^{2}} \leq\left\|\frac{1}{2} e^{-|x|}\right\|_{\mathbf{L}^{2}} \cdot\left\|u^{2}+\frac{u_{x}^{2}}{2}\right\|_{\mathbf{L}^{1}} \leq \frac{1}{\sqrt{2}} E(0) .
\end{aligned}
$$

\section{2 - An equivalent semilinear system}

Let $\bar{u} \in H^{1}(\mathbb{R})$ be an initial data. Consider an energy variable $\xi \in \mathbb{R}$, and let the nondecreasing map $\xi \mapsto \bar{y}(\xi)$ be defined by setting

$$
\int_{0}^{\bar{y}(\xi)}\left(1+\bar{u}_{x}^{2}\right) d x=\xi
$$

Assuming that the solution $u$ to the Camassa-Holm equation remains Lipschitz continuous for $t \in[0, T]$, we now derive an equivalent system of equations, using the independent variables $(t, \xi)$. Let $t \mapsto y(t, \xi)$ be the characteristic starting at $\bar{y}(\xi)$, so that

$$
\frac{\partial}{\partial t} y(t, \xi)=u(t, y(t, \xi)), \quad y(0, \xi)=\bar{y}(\xi) .
$$

Moreover, we write

$$
u(t, \xi) \doteq u(t, y(t, \xi)), \quad P(t, \xi) \doteq P(t, y(t, \xi))
$$

The following further variables will be used: $v=v(t, \xi)$ and $q=q(t, \xi)$ defined as

$$
v \doteq 2 \arctan u_{x}, \quad q \doteq\left(1+u_{x}^{2}\right) \cdot \frac{\partial y}{\partial \xi},
$$

with $u_{x}=u_{x}(t, y(t, \xi))$. We stress that $v$ is defined up to multiples of $2 \pi$. All subsequent equations involving $v$ are invariant under addition of multiples of $2 \pi$. Notice that (2.1) implies

$$
q(0, \xi) \equiv 1
$$

For future use, we write the identities

$$
\begin{aligned}
& \frac{1}{1+u_{x}^{2}}=\cos ^{2} \frac{v}{2}, \quad \frac{u_{x}}{1+u_{x}^{2}}=\frac{1}{2} \sin v, \quad \frac{u_{x}^{2}}{1+u_{x}^{2}}=\sin ^{2} \frac{v}{2}, \\
& \frac{\partial y}{\partial \xi}=\frac{q}{1+u_{x}^{2}}=\cos ^{2} \frac{v}{2} \cdot q
\end{aligned}
$$


In turn, (2.6) yields

$$
y\left(t, \xi^{\prime}\right)-y(t, \xi)=\int_{\xi}^{\xi^{\prime}} \cos ^{2} \frac{v(t, s)}{2} \cdot q(t, s) d s .
$$

Furthermore, we have

$$
\begin{gathered}
P(t, \xi)=P(t, y(t, \xi))=\frac{1}{2} \int_{-\infty}^{\infty} e^{-|y(t, \xi)-x|}\left(u^{2}(t, x)+\frac{1}{2} u_{x}^{2}(t, x)\right) d x, \\
P_{x}(t, \xi)=P_{x}(t, y(t, \xi))=\frac{1}{2}\left(\int_{y(t, \xi)}^{\infty}-\int_{-\infty}^{y(t, \xi)}\right) e^{-|y(t, \xi)-x|}\left(u^{2}(t, x)+\frac{1}{2} u_{x}^{2}(t, x)\right) d x .
\end{gathered}
$$

In the above formulas, we can perform the change of variables $x=y\left(t, \xi^{\prime}\right)$, the validity of which will be checked below (see Section 4), and write the convolution as an integral over the variable $\xi^{\prime}$. Using the identities (2.5)-(2.7), we thus obtain an expression for $P$ and $P_{x}$ in terms of the new variable $\xi$, namely

$$
\begin{aligned}
& P(\xi)=\frac{1}{2} \int_{-\infty}^{\infty} \exp \left\{-\left|\int_{\xi}^{\xi^{\prime}} \cos ^{2} \frac{v(s)}{2} \cdot q(s) d s\right|\right\} {\left[u^{2}\left(\xi^{\prime}\right) \cos ^{2} \frac{v\left(\xi^{\prime}\right)}{2}+\frac{1}{2} \sin ^{2} \frac{v\left(\xi^{\prime}\right)}{2}\right] q\left(\xi^{\prime}\right) d \xi^{\prime}, } \\
& P_{x}(\xi)=\frac{1}{2}\left(\int_{\xi}^{\infty}-\int_{-\infty}^{\xi}\right) \exp \left\{-\left|\int_{\xi}^{\xi^{\prime}} \cos ^{2} \frac{v(s)}{2} \cdot q(s) d s\right|\right\} \\
& \cdot\left[u^{2}\left(\xi^{\prime}\right) \cos ^{2} \frac{v\left(\xi^{\prime}\right)}{2}+\frac{1}{2} \sin ^{2} \frac{v\left(\xi^{\prime}\right)}{2}\right] q\left(\xi^{\prime}\right) d \xi^{\prime} .
\end{aligned}
$$

By (1.1) and (2.2), the evolution equation for $u$ in the new variables $(t, \xi)$ takes the form

$$
\frac{\partial}{\partial t} u(t, \xi)=u_{t}+u u_{x}=-P_{x}(t, \xi)
$$

with $P_{x}$ given at $(2.9)$.

To derive an evolution equation for the variable $q$ in (2.3), we observe that

$$
\int_{\xi_{1}}^{\xi_{2}} q(t, \xi) d \xi=\int_{y\left(t, \xi_{1}\right)}^{y\left(t, \xi_{2}\right)}\left(1+u_{x}^{2}(t, x)\right) d x
$$

Hence (2.2) and (1.6) yield

$$
\frac{d}{d t} \int_{\xi_{1}}^{\xi_{2}} q(t, \xi) d \xi=\int_{y\left(t, \xi_{1}\right)}^{y\left(t, \xi_{2}\right)}\left\{\left(1+u_{x}^{2}\right)_{t}+\left[u\left(1+u_{x}^{2}\right)\right]_{x}\right\} d x=\int_{y\left(t, \xi_{1}\right)}^{y\left(t, \xi_{2}\right)}\left(2 u^{2}+1-2 P\right) u_{x} d x .
$$

Differentiating w.r.t. $\xi$ we find

$$
\frac{\partial}{\partial t} q(t, \xi)=\left(2 u^{2}+1-2 P\right) \frac{u_{x}}{1+u_{x}^{2}} \cdot q=\left(u^{2}+\frac{1}{2}-P\right) \sin v \cdot q .
$$


Finally, using (2.2)-(2.3) and (1.4), we obtain

$$
\frac{\partial}{\partial t} v(t, \xi)=\frac{2}{1+u_{x}^{2}}\left[-\frac{u_{x}^{2}}{2}+u^{2}-P\right]=2\left(u^{2}-P\right) \cos ^{2} \frac{v}{2}-\sin ^{2} \frac{v}{2} .
$$

In (2.11) and in (2.12), the function $P$ is computed by (2.8).

\section{3 - Global solutions of the semilinear system}

Let an initial data $\bar{u} \in H^{1}(\mathbb{R})$ be given. We rewrite the corresponding Cauchy problem for the variables $(u, v, q)$ in the form

$$
\left\{\begin{array}{l}
\frac{\partial u}{\partial t}=-P_{x} \\
\frac{\partial v}{\partial t}=\left(u^{2}-P\right)(1+\cos v)-\sin ^{2} \frac{v}{2} \\
\frac{\partial q}{\partial t}=\left(u^{2}+\frac{1}{2}-P\right) \sin v \cdot q
\end{array}\right.
$$

with

$$
\left\{\begin{array}{l}
u(0, \xi)=\bar{u}(\bar{y}(\xi)) \\
v(0, \xi)=2 \arctan \bar{u}_{x}(\bar{y}(\xi)) \\
q(0, \xi)=1 .
\end{array}\right.
$$

Here $P, P_{x}$ are given by (2.8)-(2.9) as functions of $u, v, q$ and $\xi$. We regard (3.1) as an O.D.E. in the Banach space

$$
X \doteq H^{1}(\mathbb{R}) \times\left[\mathbf{L}^{2}(\mathbb{R}) \cap \mathbf{L}^{\infty}(\mathbb{R})\right] \times \mathbf{L}^{\infty}(\mathbb{R}),
$$

with norm

$$
\|(u, v, q)\|_{X} \doteq\|u\|_{H^{1}}+\|v\|_{\mathbf{L}^{2}}+\|v\|_{\mathbf{L}^{\infty}}+\|q\|_{\mathbf{L}^{\infty}}
$$

As usual, by a solution of the Cauchy problem we mean a fixed point of the integral transformation: $\mathcal{T}(u, v, q)=(\tilde{u}, \tilde{v}, \tilde{q})$, where

$$
\left\{\begin{array}{l}
\tilde{u}(t, \xi)=\bar{u}(\bar{y}(\xi))-\int_{0}^{t} P_{x}(\tau, \xi) d \tau \\
\tilde{v}(t, \xi)=2 \arctan \bar{u}_{x}(\bar{y}(\xi))+\int_{0}^{t}\left[\left(u^{2}-P\right)(1+\cos v)-\sin ^{2} \frac{v}{2}\right] d \tau \\
\tilde{q}(t, \xi)=1+\int_{0}^{t}\left(u^{2}+\frac{1}{2}-P\right) \sin v \cdot q d \tau
\end{array}\right.
$$

We remark that as initial data for $v$ we choose $2 \arctan u_{x} \in[-\pi, \pi]$. However, we allow $v(t, \xi) \notin$ $[-\pi, \pi]$ for $t \neq 0$. In any case, all occurrences of $v$ on the right hand side of (3.1) are clearly invariant under addition of multiples of $2 \pi$.

By proving that all functions on the right hand side of (3.1) are locally Lipschitz continuous, the local existence of solutions will follow from the standard theory of O.D.E's in Banach spaces. In a second step, exploiting the conservation of energy property expressed by (1.7), we will then prove that this local solution can be extended globally in time. 
Theorem 1. Let $\bar{u} \in H^{1}(\mathbb{R})$. Then the Cauchy problem (3.1)-(3.2) has a unique solution, defined for all times $t \in \mathbb{R}$.

Proof. Step I - Local existence. To establish the local existence of solutions, it suffices to show that the map

$$
(u, v, q) \mapsto\left(-P_{x}, \quad\left(u^{2}-P\right)(1+\cos v)-\sin ^{2} \frac{v}{2}, \quad\left(u^{2}+\frac{1}{2}-P\right) \sin v \cdot q\right),
$$

determined by the right hand side of (3.1), is Lipschitz continuous on every bounded domain $\Omega \subset X$ of the form

$$
\Omega=\left\{(u, v, q) ; \quad\|u\|_{H^{1}} \leq \alpha, \quad\|v\|_{\mathbf{L}^{2}} \leq \beta, \quad\|v\|_{\mathbf{L}^{\infty}} \leq \frac{3 \pi}{2}, \quad q(x) \in\left[q^{-}, q^{+}\right] \quad \text { for a.e. } x \in \mathbb{R}\right\},
$$

for any constants $\alpha, \beta, q^{-}, q^{+}>0$.

Due to the Sobolev's inequality

$$
\|u\|_{\mathbf{L}^{\infty}} \leq\|u\|_{H^{1}}
$$

and the uniform bounds on $v, q$, it is clear that the maps

$$
u^{2}, u^{2} \cos v, \sin ^{2} \frac{v}{2}, \sin v \cdot q,
$$

are all Lipschitz continuous as maps from $\Omega$ into $\mathbf{L}^{2}(\mathbb{R})$, and also from $\Omega$ into $\mathbf{L}^{\infty}(\mathbb{R})$.

Our main task is to prove the Lipschitz continuity of the maps

$$
(u, v, q) \mapsto P, \quad(u, v, q) \mapsto P_{x}
$$

defined at (2.8)-(2.9), as maps from $\Omega$ into $H^{1}(\mathbb{R})$. Of course, this will also imply the Lipschitz continuity of these maps from $\Omega$ into $\mathbf{L}^{2}(\mathbb{R}) \cap \mathbf{L}^{\infty}(\mathbb{R})$. Toward this goal, we first observe that, as long as $|v| \leq 3 \pi / 2$, there holds

$$
\sin ^{2} \frac{v}{2} \leq \frac{v^{2}}{4} \leq \frac{9 \pi^{2}}{8} \sin ^{2} \frac{v}{2}
$$

For $(u, v, q) \in \Omega$, by (3.6) and (3.9) one has

$$
\begin{aligned}
\text { meas }\{\xi & \left.\in \mathbb{R} ;\left|\frac{v(\xi)}{2}\right| \geq \frac{\pi}{4}\right\} \leq \text { meas }\left\{\xi \in \mathbb{R} ; \sin ^{2} \frac{v(\xi)}{2} \geq \frac{1}{18}\right\} \\
& \leq 18 \int_{\left\{\xi \in \mathbb{R}: \sin ^{2} \frac{v(\xi)}{2} \geq \frac{1}{18}\right\}} \sin ^{2} \frac{v(\xi)}{2} d \xi \leq \frac{9}{2} \beta^{2} .
\end{aligned}
$$

Therefore, using again (3.6), for any $\xi_{1}<\xi_{2}$ we find

$$
\int_{\xi_{1}}^{\xi_{2}} \cos ^{2} \frac{v(\xi)}{2} \cdot q\left(\xi^{\prime}\right) d \xi \geq \int_{\left\{\xi \in\left[\xi_{1}, \xi_{2}\right] ;\left|\frac{v(\xi)}{2}\right| \leq \frac{\pi}{4}\right\}} \frac{q^{-}}{2} d \xi \geq\left[\frac{\xi_{2}-\xi_{1}}{2}-\frac{9}{2} \beta^{2}\right] q^{-} .
$$

The above is a key estimate, which guarantees that the exponential term in the formulas (2.8)-(2.9) for $P$ and $P_{x}$ decreases quickly as $\left|\xi-\xi^{\prime}\right| \rightarrow \infty$. Introducing the exponentially decaying function

$$
\Gamma(\zeta) \doteq \min \left\{1, \exp \left(\frac{9}{2} \beta^{2} q^{-}-\frac{|\zeta|}{2} q^{-}\right)\right\}
$$


an easy computation shows that

$$
\|\Gamma\|_{\mathbf{L}^{1}}=\left(\int_{|\zeta| \leq 9 \beta^{2}}+\int_{|\zeta| \geq 9 \beta^{2}}\right) \Gamma(\zeta) d \zeta=18 \beta^{2}+\frac{4}{q^{-}} .
$$

We begin by showing that $P, P_{x} \in H^{1}(\mathbb{R})$, namely

$$
P, \quad \partial_{\xi} P, \quad P_{x}, \quad \partial_{\xi} P_{x} \in \mathbf{L}^{2}(\mathbb{R}) .
$$

In the following we derive a priori bounds on $P_{x}$. The estimates for $P$ are entirely similar. From the definition (2.9) it follows

$$
\left|P_{x}(\xi)\right| \leq \frac{q^{+}}{2}\left|\Gamma *\left(u^{2} \cos ^{2} \frac{v}{2}+\frac{1}{2} \sin ^{2} \frac{v}{2}\right)(\xi)\right| .
$$

Therefore, using standard properties of convolutions we obtain

$$
\begin{aligned}
\left\|P_{x}\right\|_{\mathbf{L}^{2}} & \leq \frac{q^{+}}{2} \cdot\|\Gamma\|_{\mathbf{L}^{1}} \cdot\left(\left\|u^{2}\right\|_{\mathbf{L}^{2}}+\frac{1}{8}\left\|v^{2}\right\|_{\mathbf{L}^{2}}\right) \\
& \leq \frac{q^{+}}{2} \cdot\|\Gamma\|_{\mathbf{L}^{1}} \cdot\left(\|u\|_{\mathbf{L}^{\infty}}^{2}\left\|u^{2}\right\|_{\mathbf{L}^{2}}+\frac{1}{8}\|v\|_{\mathbf{L}^{\infty}}^{2}\left\|v^{2}\right\|_{\mathbf{L}^{2}}\right)<\infty .
\end{aligned}
$$

Next, differentiating (2.9) we find

$$
\begin{aligned}
\frac{\partial}{\partial \xi} P_{x}(\xi) & =-\left[u^{2}(\xi) \cos ^{2} \frac{v(\xi)}{2}+\frac{1}{2} \sin ^{2} \frac{v(\xi)}{2}\right] q(\xi) \\
+\frac{1}{2} & \left(\int_{\xi}^{\infty}-\int_{-\infty}^{\xi}\right) \exp \left\{-\left|\int_{\xi}^{\xi^{\prime}} \cos ^{2} \frac{v(s)}{2} \cdot q(s) d s\right|\right\} \\
\cdot & {\left[\cos ^{2} \frac{v(\xi)}{2} \cdot q(\xi)\right] \operatorname{sign}\left(\xi^{\prime}-\xi\right)\left[u^{2}\left(\xi^{\prime}\right) \cos ^{2} \frac{v\left(\xi^{\prime}\right)}{2}+\frac{1}{2} \sin ^{2} \frac{v\left(\xi^{\prime}\right)}{2}\right] q\left(\xi^{\prime}\right) d \xi^{\prime} . }
\end{aligned}
$$

Therefore

$$
\begin{aligned}
\left|\partial_{\xi} P_{x}(\xi)\right| & \leq q^{+}\left|u^{2}(\xi)+\frac{v^{2}(\xi)}{8}\right|+\frac{q^{+}}{2}\left|\Gamma *\left(u^{2} \cos ^{2} \frac{v}{2}+\frac{1}{2} \sin ^{2} \frac{v}{2}\right)(\xi)\right| \\
\left\|\partial_{\xi} P_{x}\right\|_{\mathbf{L}^{2}} & \leq q^{+}\left(\left\|u^{2}\right\|_{\mathbf{L}^{2}}+\frac{1}{8}\left\|v^{2}\right\|_{\mathbf{L}^{2}}\right)+\frac{q^{+}}{2} \cdot\|\Gamma\|_{\mathbf{L}^{1}} \cdot\left(\left\|u^{2}\right\|_{\mathbf{L}^{2}}+\frac{1}{8}\left\|v^{2}\right\|_{\mathbf{L}^{2}}\right) \\
& \leq\left(q^{+}+\frac{q^{+}}{2}\|\Gamma\|_{\mathbf{L}^{1}}\right) \cdot\left(\|u\|_{\mathbf{L}^{\infty}}^{2}\|u\|_{\mathbf{L}^{2}}+\frac{1}{8}\|v\|_{\mathbf{L}^{\infty}}^{2}\|v\|_{\mathbf{L}^{2}}\right)<\infty .
\end{aligned}
$$

By (3.14), (3.16) and the analogous estimates for $P, \partial_{\xi} P$, relation (3.13) follows.

We just proved that the maps in (3.8) actually take values in $H^{1}(\mathbb{R})$. To establish their Lipschitz continuity, it suffices to show that their partial derivatives

$$
\frac{\partial P}{\partial u}, \frac{\partial P}{\partial v}, \frac{\partial P}{\partial q}, \frac{\partial P_{x}}{\partial u}, \frac{\partial P_{x}}{\partial v}, \frac{\partial P_{x}}{\partial q},
$$

are uniformly bounded as $(u, v, q)$ range inside the domain $\Omega$. We observe that these derivatives are bounded linear operators from the appropriate spaces into $H^{1}(\mathbb{R})$. For sake of illustration, we 
shall work out the detailed estimates for $\partial P_{x} / \partial u$. All other derivatives can be estimated by the same methods.

At a given point $(u, v, q) \in \Omega$, the partial derivative $\partial P / \partial u: H^{1}(\mathbb{R}) \mapsto \mathbf{L}^{2}(\mathbb{R})$ is the linear operator defined by

$$
\begin{aligned}
& {\left[\frac{\partial P_{x}(u, v, q)}{\partial u} \cdot \hat{u}\right](\xi)} \\
& =\frac{1}{2}\left(\int_{\xi}^{\infty}-\int_{-\infty}^{\xi}\right) \exp \left\{-\left|\int_{\xi}^{\xi^{\prime}} \cos ^{2} \frac{v(s)}{2} \cdot q(s) d s\right|\right\} \cdot 2 u\left(\xi^{\prime}\right) \cos ^{2} \frac{v\left(\xi^{\prime}\right)}{2} q\left(\xi^{\prime}\right) \cdot \hat{u}\left(\xi^{\prime}\right) d \xi^{\prime}
\end{aligned}
$$

Therefore

$$
\left\|\frac{\partial P_{x}(u, v, q)}{\partial u} \cdot \hat{u}\right\|_{\mathbf{L}^{2}} \leq\|\Gamma *|u|\|_{\mathbf{L}^{2}} q^{+} \cdot\|\hat{u}\|_{\mathbf{L}^{\infty}} .
$$

Recalling that $\|\hat{u}\|_{\mathbf{L}^{\infty}} \leq\|\hat{u}\|_{H^{1}}$, the operator norm can thus be estimated as

$$
\left\|\frac{\partial P_{x}(u, v, q)}{\partial u}\right\| \leq q^{+}\|\Gamma\|_{\mathbf{L}^{1}} \cdot\|u\|_{\mathbf{L}^{2}} .
$$

Next, by (3.15), $\partial\left(\partial_{\xi} P_{x}\right) / \partial u: H^{1}(\mathbb{R}) \mapsto \mathbf{L}^{2}(\mathbb{R})$ is the linear operator defined by

$$
\begin{aligned}
& \quad\left[\frac{\partial\left(\partial_{\xi} P_{x}\right)(u, v, q)}{\partial u} \cdot \hat{u}\right](\xi) \\
& =-2 u(\xi) \cos ^{2} \frac{v(\xi)}{2} q(\xi) \cdot \hat{u}(\xi)+\frac{1}{2}\left(\int_{\xi}^{\infty}-\int_{-\infty}^{\xi}\right) \exp \left\{-\left|\int_{\xi}^{\xi^{\prime}} \cos ^{2} \frac{v(s)}{2} \cdot q(s) d s\right|\right\} \\
& \quad \cdot\left[\cos ^{2} \frac{v(\xi)}{2} \cdot q(\xi)\right] \operatorname{sign}\left(\xi^{\prime}-\xi\right) 2 u\left(\xi^{\prime}\right) \cos ^{2} \frac{v\left(\xi^{\prime}\right)}{2} q\left(\xi^{\prime}\right) \cdot \hat{u}\left(\xi^{\prime}\right) d \xi^{\prime} .
\end{aligned}
$$

Its norm, as an operator from $H^{1}(\mathbb{R})$ into $\mathbf{L}^{2}(\mathbb{R})$, is thus bounded by

$$
\left\|\frac{\partial\left(\partial_{\xi} P_{x}\right)(u, v, q)}{\partial u}\right\| \leq 2 q^{+}\|u\|_{\mathbf{L}^{2}}+\left(q^{+}\right)^{2}\|\Gamma\|_{\mathbf{L}^{1}} \cdot\|u\|_{\mathbf{L}^{2}} .
$$

Together, (3.18) and (3.19) yield the boundedness of $\partial P_{x} / \partial u$ as a linear operator from $H^{1}(\mathbb{R})$ into $H^{1}(\mathbb{R})$. The bounds on the other partial derivatives in (3.17) are obtained in an entirely similar way. In the end, this establishes the uniform Lipschitz continuity of the maps $P, P_{x}$ at (3.8).

The local existence of a solution to the Cauchy problem (3.1)-(3.2) on some small time interval $[-T, T]$ now follows from the standard theory of O.D.E's in Banach spaces. Indeed, we proved that the right hand side of (3.1) is Lipschitz continuous on a neighborhood of the initial data, in the space $X$.

Step II - Extension to a global solution. To ensure that the local solution of (3.1) constructed above can be extended to a global solution defined for all $t \geq 0$, it suffices to show that the quantity

$$
\|q(t)\|_{\mathbf{L}^{\infty}}+\left\|\frac{1}{q(t)}\right\|_{\mathbf{L}^{\infty}}+\|v(t)\|_{\mathbf{L}^{2}}+\|v(t)\|_{\mathbf{L}^{\infty}}+\|u(t)\|_{H^{1}}
$$


remains uniformly bounded on any bounded time interval. The a priori bound on (3.20) will actually follow from the conservation of the total energy (1.7). In the following, we re-derive this energy conservation property in terms of the new variables $(u, v, q)$ and $\xi$.

As long as the local solution of (3.1) is defined, we claim that

$$
u_{\xi}=\frac{q}{2} \sin v
$$

and

$$
\frac{d}{d t} \int_{\mathbb{R}}\left(u^{2} \cos ^{2} \frac{v}{2}+\sin ^{2} \frac{v}{2}\right) q d \xi=0 .
$$

Indeed, (3.1) yields

$$
u_{\xi t}=q\left(u^{2} \cos ^{2} \frac{v}{2}+\frac{1}{2} \sin ^{2} \frac{v}{2}-P \cos ^{2} \frac{v}{2}\right)
$$

if we take into account (2.8)-(2.9). On the other hand, by (3.1) we get

$$
\begin{aligned}
\left(\frac{q}{2} \sin v\right)_{t} & =\frac{q_{t}}{2} \sin v+\frac{q}{2} v_{t} \cos v \\
& =\frac{q}{2}\left(\left(u^{2}+\frac{1}{2}-P\right) \sin ^{2} v+\left(u^{2}-P\right) \cos v+\left(u^{2}-P\right) \cos ^{2} v-\cos v \sin ^{2} \frac{v}{2}\right) \\
& =q\left(u^{2} \cos ^{2} \frac{v}{2}+\frac{1}{2} \sin ^{2} \frac{v}{2}-P \cos ^{2} \frac{v}{2}\right) .
\end{aligned}
$$

Moreover, at the initial time $t=0$, by (2.5) and (3.2) we have

$$
\frac{\partial u}{\partial \xi}=\frac{u_{x}}{1+u_{x}^{2}}=\frac{\sin v}{2}, \quad q \equiv 1 .
$$

Since the right hand sides of (3.23)-(3.24) are Lipschitz continuous and the identity (3.21) holds at $t=0$, we infer that (3.21) remains valid for all times $t$, as long as the solution is defined.

To prove (3.22), we proceed as follows. From (3.1) we deduce that

$$
\begin{aligned}
& \frac{d}{d t} \int_{\mathbb{R}}\left(u^{2} \cos ^{2} \frac{v}{2}+\sin ^{2} \frac{v}{2}\right) q d \xi=\int_{\mathbb{R}} q\left\{2\left(u^{2} \cos ^{2} \frac{v}{2}+\sin ^{2} \frac{v}{2}\right)\left(u^{2}+\frac{1}{2}-P\right) \sin \frac{v}{2} \cos \frac{v}{2}\right. \\
& \left.-2 u P_{x} \cos ^{2} \frac{v}{2}+\sin \frac{v}{2} \cos \frac{v}{2}\left(1-u^{2}\right)\left[2\left(u^{2}-P\right) \cos ^{2} \frac{v}{2}-\sin ^{2} \frac{v}{2}\right]\right\} d \xi \\
& =\int_{\mathbb{R}} q\left\{-2 P \sin \frac{v}{2} \cos \frac{v}{2}-2 u P_{x} \cos ^{2} \frac{v}{2}+3 u^{2} \sin \frac{v}{2} \cos \frac{v}{2}\right\} d \xi .
\end{aligned}
$$

On the other hand, from (2.8)-(2.9) we infer

$$
P_{\xi}=q P_{x} \cos ^{2} \frac{v}{2} .
$$

Therefore, using (3.21) we obtain

$$
(u P)_{\xi}=u_{\xi} P+u P_{\xi}=q\left\{P \sin \frac{v}{2} \cos \frac{v}{2}+u P_{x} \cos ^{2} \frac{v}{2}\right\}
$$

while

$$
3 q u^{2} \sin \frac{v}{2} \cos \frac{v}{2}=3 u^{2} u_{\xi}=\left(u^{3}\right)_{\xi} .
$$


The above implies

$$
\frac{d}{d t} \int_{\mathbb{R}}\left(u^{2} \cos ^{2} \frac{v}{2}+\sin ^{2} \frac{v}{2}\right) q d \xi=\int_{\mathbb{R}} \partial_{\xi}\left\{u^{3}-2 u P\right\} d \xi=0,
$$

the last equality being justified since $P$ is uniformly bounded while $\lim _{|\xi| \rightarrow \infty} u(\xi)=0$ as $u \in$ $H^{1}(\mathbb{R})$. This proves $(3.22)$.

We can now rewrite the total energy (1.7) in terms of the new variables According to (3.22), this energy remains constant in time, along any solution of (3.1)-(3.2):

$$
E(t)=\int_{\mathbb{R}}\left(u^{2}(t, \xi) \cos ^{2} \frac{v(t, \xi)}{2}+\sin ^{2} \frac{v(t, \xi)}{2}\right) q(t, \xi) d \xi=E(0) \doteq E_{0}
$$

As long as the solution is defined, using (3.21) and (3.26) we obtain the bound

$$
\sup _{\xi \in \mathbb{R}}\left|u^{2}(t, \xi)\right| \leq 2 \int_{\mathbb{R}}\left|u u_{\xi}\right| d \xi \leq 2 \int_{\mathbb{R}}|u|\left|\sin \frac{v}{2} \cos \frac{v}{2}\right| q d \xi \leq E_{0}
$$

This provides a uniform a priori bound on $\|u(t)\|_{\mathbf{L}^{\infty}}$. From (3.26) and the definitions (2.8)-(2.9) it follows

$$
\|P(t)\|_{\mathbf{L}^{\infty}}, \quad\left\|P_{x}(t)\right\|_{\mathbf{L}^{\infty}} \leq\left\|\frac{1}{2} e^{-|x|}\right\|_{\mathbf{L}^{\infty}} \cdot\left\|u^{2}(t)+\frac{u_{x}^{2}(t)}{2}\right\|_{\mathbf{L}^{1}} \leq \frac{1}{2} E_{0} .
$$

We thus recover the estimate (1.8), working in the new variables.

Looking at the third equation in (3.1), by (3.27)-(3.28) we deduce that, as long as the solution is defined,

$$
\left|q_{t}\right| \leq\left(E_{0}+\frac{1}{2}+\frac{E_{0}}{2}\right) q .
$$

Since $q(0, \xi)=1$, the previous differential inequality yields

$$
\exp \left\{-\frac{1+3 E_{0}}{2}|t|\right\} \leq q(t) \leq \exp \left\{\frac{1+3 E_{0}}{2}|t|\right\}
$$

By the second equation in (3.1), it is now clear that

$$
\|v(t)\|_{\mathbf{L}^{\infty}} \leq e^{B|t|},
$$

for a suitable constant $B=B\left(E_{0}\right)>0$. Moreover, the first equation in (3.1) implies

$$
\begin{gathered}
\left|\frac{d}{d t}\left(\int_{\mathbb{R}} u^{2}(t, \xi) d \xi\right)\right| \leq 2\|u(t)\|_{\mathbf{L}^{\infty}}\left\|P_{x}(t)\right\|_{\mathbf{L}^{1}} . \\
\left|\frac{d}{d t}\left(\int_{\mathbb{R}} u_{\xi}^{2}(t, \xi) d \xi\right)\right| \leq 2\left\|u_{\xi}(t)\right\|_{\mathbf{L}^{\infty}}\left\|\partial_{\xi} P_{x}(t)\right\|_{\mathbf{L}^{1}} .
\end{gathered}
$$

We already proved that the functions $u$ and $u_{\xi}$ are uniformly bounded on bounded intervals of time, because of (3.27), (3.21), and (3.29). An estimate on $\|u(t)\|_{H^{1}}$ will thus follow from a bound 
on the $\mathbf{L}^{1}$ norms of $P_{x}$ and $\partial_{\xi} P_{x}$. Toward this goal, we proceed as in (3.9)-(3.12). Calling $\kappa$ the right hand side of (3.29), so that $\kappa^{-1} \leq q(t) \leq \kappa$, from (3.15) we deduce

$$
\begin{aligned}
& \left\|\partial_{\xi} P_{x}(t)\right\|_{\mathbf{L}^{1}} \leq \kappa E_{0} \\
& +\frac{1}{2} \int_{\mathbb{R}} \exp \left\{-\left|\int_{\xi}^{\xi^{\prime}} \kappa^{-1} \cos ^{2} \frac{v(t, s)}{2} d s\right|\right\} \cdot\left[u^{2}\left(t, \xi^{\prime}\right) \cos ^{2} \frac{v(t, \xi)}{2}+\frac{1}{2} \sin ^{2} \frac{v(t, \xi)}{2}\right] \kappa d \xi^{\prime} \\
& \leq \kappa E_{0}+\|\Gamma\|_{\mathbf{L}^{1}} \cdot \kappa E_{0} .
\end{aligned}
$$

where

$$
\Gamma(\zeta) \doteq \min \left\{1, \exp \left(18 E_{0} \kappa^{-1}-\frac{|\zeta|}{2} \kappa^{-1}\right)\right\}
$$

Indeed, by (3.26), the integral of $\sin ^{2}(v / 2)$ is bounded uniformly in time. We can thus repeat the estimates in (3.10)-(3.12) and deduce

$$
\|\Gamma\|_{\mathbf{L}^{1}}=\left(\int_{|\zeta| \leq 36 E_{0}}+\int_{|\zeta| \geq 36 E_{0}}\right) \Gamma(\zeta) d \zeta=72 E_{0}+\frac{4}{\kappa} .
$$

The estimate for $\|P\|_{\mathbf{L}^{1}}$ is entirely similar. This establishes the boundedness of the norm $\|u(t)\|_{H^{1}}$ for $t$ in bounded intervals.

Finally, the second equation in (3.1) implies

$$
\frac{d}{d t}\|v\|_{\mathbf{L}^{2}} \leq 2\left(\|u\|_{\mathbf{L}^{\infty}}\|u\|_{\mathbf{L}^{2}}+\|P\|_{\mathbf{L}^{2}}\right)+\frac{1}{4}\|v\|_{\mathbf{L}^{\infty}}\|v\|_{\mathbf{L}^{2}} .
$$

By the previous bounds, it is clear that $\|v\|_{\mathbf{L}^{2}}$ remains bounded on bounded intervals of time. This completes the proof that the solution of (3.1) can be extended globally in time.

For future use, we record here an important property of the above solutions. Namely, consider the set of times

$$
\mathcal{N} \doteq\{t \geq 0 ; \quad \operatorname{meas}\{\xi \in \mathbb{R} ; v(t, \xi)=-\pi\}>0\}
$$

Then

$$
\operatorname{meas}(\mathcal{N})=0 .
$$

Indeed, when $\cos v=-1$, by (3.1) it follows $v_{t}=-1$. Using the bounds on $\|u\|_{\mathbf{L}^{\infty}}$ and $\left\|P_{x}\right\|_{\mathbf{L}^{\infty}}$, on any bounded time interval we can find $\delta>0$ such that $v_{t}<-1 / 2$ whenever $1+\cos v(t, x)<\delta$. Since $\|v(t)\|_{\mathbf{L}^{2}}$ remains bounded on bounded time-intervals, this transversality condition implies (3.32) as otherwise we would have that $\iint_{\{v(t, \xi)=-\pi\}} v_{t} d \xi d t<0$ which is impossible since $v_{t}=0$ a.e. on $\{v(t, \xi)=-\pi\}$ due to the absolute continuity of the map $t \mapsto v(t, \xi)$ at every fixed $\xi \in \mathbb{R}$.

\section{4 - Solutions to the Camassa-Holm equation}

We now show that the global solution of the system (3.1) yields a global conservative solution to the Camassa-Holm equation (1.1), in the original variables $(t, x)$.

Let us start with a global solution $(u, v, q)$ to (3.1). Define

$$
y(t, \xi) \doteq \bar{y}(\xi)+\int_{0}^{t} u(\tau, \xi) d \tau .
$$


For each fixed $\xi$, the function $t \mapsto y(t, \xi)$ thus provides a solution to the Cauchy problem

$$
\frac{\partial}{\partial t} y(t, \xi)=u(t, \xi), \quad y(0, \xi)=\bar{y}(\xi) .
$$

We claim that a solution of (1.1) can be obtained by setting

$$
u(t, x) \doteq u(t, \xi) \quad \text { if } y(t, \xi)=x .
$$

Theorem 2. Let $(u, v, q)$ provide a global solution to the Cauchy problem (3.1)-(3.2). Then the function $u=u(t, x)$ defined by (4.1), (4.3) provides a solution to the initial value problem (1.1)(1.2) for the Camassa-Holm equation.

The solution $u$ constructed in this way has the following properties. The energy is almost always conserved, namely

$$
\|u(t)\|_{H^{1}(\mathbb{R})}=\|\bar{u}\|_{H^{1}(\mathbb{R})} \quad \text { for a.e. } t \in \mathbb{R} .
$$

Moreover, consider a sequence of initial data $\bar{u}_{n}$ such that $\left\|\bar{u}_{n}-\bar{u}\right\|_{H^{1}(\mathbb{R})} \rightarrow 0$. Then the corresponding solutions $u_{n}=u_{n}(t, x)$ converge to $u(t, x)$ uniformly for $t, x$ in bounded sets.

Proof. Using the uniform bound $|u(t, \xi)| \leq E_{0}^{1 / 2}$, valid by (3.27), from (4.1) we obtain the estimate

$$
\bar{y}(\xi)-E_{0}^{1 / 2} t \leq y(t, \xi) \leq \bar{y}(\xi)+E_{0}^{1 / 2} t .
$$

Recalling the definition of $\xi$ at (2.1), this yields

$$
\lim _{\xi \rightarrow \pm \infty} \bar{y}(t, \xi)= \pm \infty
$$

Therefore, the image of the continuous map $(t, \xi) \mapsto(t, y(t, \xi))$ is the entire plane $\mathbb{R}^{2}$. Next, we establish the identity

$$
y_{\xi}=q \cos ^{2} \frac{v}{2}
$$

for all $t$ and a.e. $\xi \in \mathbb{R}$. Indeed, notice that (3.1) yields

$$
\begin{aligned}
\frac{\partial}{\partial t}\left(q \cos ^{2} \frac{v}{2}\right)(t, \xi) & =-v_{t} \sin \frac{v}{2} \cos \frac{v}{2}+q_{t} \cos ^{2} \frac{v}{2} \\
& =q \cos \frac{v}{2} \sin ^{3} \frac{v}{2}-2 q\left(u^{2}-P\right) \cos ^{3} \frac{v}{2} \sin \frac{v}{2}+2\left(u^{2}+\frac{1}{2}-P\right) \sin \frac{v}{2} \cos ^{3} \frac{v}{2} \\
& =q \cos \frac{v}{2} \sin ^{3} \frac{v}{2}+q \sin \frac{v}{2} \cos ^{3} \frac{v}{2}=q \sin \frac{v}{2} \cos \frac{v}{2}=\frac{q}{2} \sin v \\
& =u_{\xi}(t, \xi),
\end{aligned}
$$

in view of (3.21). On the other hand, (4.1) implies

$$
\frac{\partial}{\partial t} y_{\xi}(t, \xi)=u_{\xi}(t, \xi) .
$$

Since the function $x \mapsto 2 \arctan \bar{u}_{x}(x)$ is measurable, the identity (4.5) holds for almost every $\xi \in \mathbb{R}$ at $t=0$. By the above computations it remains true for all times $t \in \mathbb{R}$. 
An immediate consequence of (4.5) is that the map $\xi \mapsto y(t, \xi)$ is non-decreasing. Moreover, if $\xi<\xi^{\prime}$ but $y(t, \xi)=y\left(t, \xi^{\prime}\right)$, then

$$
\int_{\xi}^{\xi^{\prime}} y_{\xi}(t, s) d s=\int_{\xi}^{\xi^{\prime}} q(t, s) \cos ^{2} \frac{v(t, s)}{2} d s=0 .
$$

Hence $\cos (v / 2) \equiv 0$ throughout the interval of integration. Therefore, by (3.21)

$$
u\left(t, \xi^{\prime}\right)-u(t, \xi)=\int_{\xi}^{\xi^{\prime}} \frac{q(t, s)}{2} \sin v(t, s) d s=0 .
$$

This proves that the the map $(t, x) \mapsto u(t, x)$ at (4.3) is well defined, for all $(t, x) \in \mathbb{R}^{2}$.

For reader's convenience, we collect here the basic relations between the $(t, x)$ and the $(t, \xi)$ variables.

$$
\begin{gathered}
\frac{\partial u}{\partial \xi}(t, \xi)=\frac{q(t, \xi)}{2} \sin v(t, \xi), \\
\frac{\partial y}{\partial \xi}(t, \xi)=q(t, \xi) \cos ^{2} \frac{v(t, \xi)}{2} \\
u_{x}(t, x)=\frac{\sin v(t, \xi)}{1+\cos v(t, \xi)} \quad \text { if } \quad x=y(t, \xi), \cos v(t, \xi) \neq-1 .
\end{gathered}
$$

Next, using (4.6) to change the variable of integration, for every fixed $t$ we compute

$$
\begin{aligned}
& \int_{\mathbb{R}}\left(u^{2}(t, x)+u_{x}^{2}(t, x)\right) d x \\
& \quad=\int_{\{\cos v>-1\}}\left(u^{2}(t, \xi) \cos ^{2} \frac{v(t, \xi)}{2}+\sin ^{2} \frac{v(t, \xi)}{2}\right) q(t, \xi) d \xi \leq E_{0},
\end{aligned}
$$

because of (3.26). By a Sobolev inequality [EG], this implies the uniform Hölder continuity with exponent $1 / 2$ of $u$ as a function of $x$. By the first equation in (3.1) and the bound $\left\|P_{x}\right\|_{\mathbf{L}^{\infty}} \leq E_{0} / 2$, it follows that the map $t \mapsto u(t, y(t))$ is uniformly Lipschitz continuous along every characteristic curve $t \mapsto y(t)$. Therefore, $u=u(t, x)$ is globally Hölder continuous on the entire $t$ - $x$ plane.

We now prove that the map $t \mapsto u(t)$ is Lipschitz continuous with values in $\mathbf{L}^{2}(\mathbb{R})$. Indeed, consider any interval $[\tau, \tau+h]$. For a given point $x$, choose $\xi \in \mathbb{R}$ such that the characteristic $t \mapsto y(t, \xi)$ passes through the point $(\tau, x)$. By (3.1) and the bound (3.27) it follows

$$
\begin{aligned}
|u(\tau+h, x)-u(\tau, x)| & \leq|u(\tau+h, x)-u(\tau+h, y(\tau+h, \xi))|+|u(\tau+h, y(\tau+h, \xi))-u(\tau, x)| \\
& \leq \sup _{|y-x| \leq E_{0}^{1 / 2} h}|u(t+h, y)-u(\tau+h, x)| d x+\int_{\tau}^{\tau+h}\left|P_{x}(t, \xi)\right| d t .
\end{aligned}
$$


Integrating over the whole real line we obtain

$$
\begin{aligned}
\int_{\mathbb{R}} & |u(\tau+h, x)-u(\tau, x)|^{2} d x \\
& \leq 2 \int_{\mathbb{R}}\left(\int_{x-E_{0}^{1 / 2} h}^{x+E_{0}^{1 / 2} h}\left|u_{x}(\tau+h, y)\right| d y\right)^{2} d x+2 \int_{\mathbb{R}}\left(\int_{\tau}^{\tau+h}\left|P_{x}(t, \xi)\right| d t\right)^{2} q(\tau, \xi) \cos ^{2} \frac{v(\tau, \xi)}{2} d \xi \\
& \leq 2 \int_{\mathbb{R}}\left(2 E_{0}^{1 / 2} h \int_{x-E_{0}^{1 / 2} h}^{x+E_{0}^{1 / 2} h}\left|u_{x}(\tau+h, y)\right|^{2} d y\right) d x+2 \int_{\mathbb{R}}\left(h \int_{\tau}^{\tau+h}\left|P_{x}(t, \xi)\right|^{2} d t\right)\|q(\tau)\|_{\mathbf{L}^{\infty} d \xi} \\
& =4 E_{0}^{1 / 2} h \int_{\mathbb{R}} \int_{y-E_{0}^{1 / 2} h}^{y+E_{0}^{1 / 2} h}\left|u_{x}(\tau+h, y)\right|^{2} d x d y+2 h\|q(\tau)\|_{\mathbf{L}^{\infty}} \int_{\mathbb{R}^{R}} \int_{\tau}^{\tau+h}\left|P_{x}(t, \xi)\right|^{2} d t d \xi \\
& \leq 8 E_{0} h^{2}\left\|u_{x}(\tau+h)\right\|_{\mathbf{L}^{2}}^{2}+2 h\|q(\tau)\|_{\mathbf{L}^{\infty}} \int_{\tau}^{\tau+h}\left\|P_{x}(t)\right\|_{\mathbf{L}^{2}}^{2} d t \\
& \leq C h^{2}
\end{aligned}
$$

for some constant $C$ uniformly valid as $t$ ranges on bounded set, in view of (3.14), (3.29) and (4.8). This clearly implies the Lipschitz continuity of the map $t \mapsto u(t)$, in terms of the $x$-variable.

Since $\mathbf{L}^{2}(\mathbb{R})$ is a reflexive space, the left hand side of (1.3) is a well defined function, for a.e. $t \in \mathbb{R}$. We already proved that the right hand side also lies in $\mathbf{L}^{2}(\mathbb{R})$ for a.e. $t \in \mathbb{R}$ in view of the infinite-dimensional version of Rademacher's theorem [A]. To establish the equality between the two sides, we observe that

$$
\frac{d}{d t} u(t, y(t, \xi))=P_{x}(t, \xi)
$$

for every $t, \xi \in \mathbb{R}$. Here $P_{x}$ is the function defined at (2.9). On the other hand, recalling (3.31), for every $t \notin \mathcal{N}$, the map $\xi \mapsto x(t, \xi)$ is one to one and the change of variable formulas (4.6)-(4.7) yield

$$
P_{x}(t, \xi)=P_{x}(t, y(t, \xi))=\frac{1}{2}\left(\int_{y(t, \xi)}^{\infty}-\int_{-\infty}^{y(t, \xi)}\right) e^{-|y(t, \xi)-x|}\left(u^{2}(t, x)+\frac{1}{2} u_{x}^{2}(t, x)\right) d x
$$

for every $\xi \in \mathbb{R}$. According to (3.32), this set $\mathcal{N}$ of exceptional times has measure zero. Hence the identity (1.3) is satisfied for almost every $t \in \mathbb{R}$. This completes the proof that $u$ is a global solution of the Camassa-Holm equation in the sense of Definition 1.

To prove the conservation property, call $\mathcal{N}$ the set of exceptional times defined at (3.31). We recall that meas $(\mathcal{N})=0$. For all $t \notin \mathcal{N}$, from (3.26) and (4.8) we obtain the identity in (4.4).

Finally, let $\bar{u}_{n}$ be a sequence of initial data converging to $\bar{u}$ in $H^{1}(\mathbb{R})$. Recalling $(2.1)$ and (3.2), at time $t=0$ this implies

$$
\sup _{\xi \in \mathbb{R}}\left|y_{n}(0, \xi)-y(0, \xi)\right| \rightarrow 0, \quad \sup _{\xi \in \mathbb{R}}\left|u_{n}(0, \xi)-u(0, \xi)\right| \rightarrow 0,
$$

Moreover,

$$
\left\|v_{n}(0, \cdot)-v(0, \cdot)\right\|_{\mathbf{L}^{2}} \rightarrow 0 .
$$

This implies $u_{n}(t, \xi) \rightarrow u(t, \xi)$, uniformly for $t, \xi$ in bounded sets. Returning to the original coordinates, this yields the convergence

$$
y_{n}(t, \xi) \rightarrow y(t, \xi), \quad u(t, x) \rightarrow u_{n}(t, x),
$$


uniformly on bounded sets, because all functions $u, u_{n}$ are uniformly Hölder continuous.

\section{5 - A semigroup of conservative solutions}

In the previous sections, for each initial data $\bar{u} \in H^{1}(\mathbb{R})$, we constructed a global conservative solution to the Camassa-Holm equation. We remark, however, that the resulting flow $u(t)=\Psi_{t} \bar{u}$ is not yet a semigroup. Indeed, if $t \in \mathcal{N}$, the semigroup property fails.

Example (Peakon-antipeakon interaction). Motivated by the form of the peakons, it is natural to make the following Ansatz $[\mathrm{CH}]$ for the interaction of two solitary waves

$$
u(t, x)=p_{1}(t) e^{-\left|x-q_{1}(t)\right|}+p_{2}(t) e^{-\left|x-q_{2}(t)\right|}, \quad x \in \mathbb{R}, \quad t \geq 0,
$$

with $p_{1}, q_{1}, p_{2}, q_{2}$ Lipschitz continuous such that $q_{1}(0) \neq q_{2}(0)$. Notice that (5.1) is a solution in the sense of Definition 1 if the variables $q_{1}(t), q_{2}(t), p_{1}(t), p_{2}(t)$ satisfy the following system of ordinary differential equations with discontinuous right-hand side

$$
\left\{\begin{array}{l}
q_{1}^{\prime}=p_{1}+p_{2} e^{-\left|q_{1}-q_{2}\right|} \\
q_{2}^{\prime}=p_{1} e^{-\left|q_{1}-q_{2}\right|}+p_{2} \\
p_{1}^{\prime}=p_{1} p_{2} \operatorname{sign}\left(q_{1}-q_{2}\right) e^{-\left|q_{1}-q_{2}\right|} \\
p_{2}^{\prime}=p_{1} p_{2} \operatorname{sign}\left(q_{2}-q_{1}\right) e^{-\left|q_{1}-q_{2}\right|}
\end{array}\right.
$$

interpreted in the sense of Carathéodory (see [CE1]). Observe that (5.2) is a Hamiltonian system with Hamiltonian

$$
H=\frac{1}{2} \sum_{i, j=1,2} p_{i} p_{j} e^{-\left|q_{i}-q_{j}\right|}
$$

and, in addition to $H$, the system (5.2) admits the conserved quantity $p_{1}+p_{2}$ as long as $q_{1}-q_{2} \neq 0$, relation which by continuity will hold true at least for $t>0$ small. These observations motivate the change to the new canonical variables

$$
\left\{\begin{aligned}
P=p_{1}+p_{2}, & Q=q_{1}+q_{2}, \\
p=p_{1}-p_{2}, & q=q_{1}-q_{2},
\end{aligned}\right.
$$

in terms of which (5.2) becomes the Hamiltonian system

$$
\left\{\begin{array}{l}
P^{\prime}=0 \\
Q^{\prime}=P\left(1+e^{-|q|}\right), \\
p^{\prime}=\frac{P^{2}-p^{2}}{2} \operatorname{sign}(q) e^{-|q|}, \\
q^{\prime}=p\left(1-e^{-|q|}\right),
\end{array}\right.
$$

with Hamiltonian

$$
\tilde{H}=\frac{1}{2} P^{2}\left(1+e^{-|q|}\right)+\frac{1}{2} p^{2}\left(1-e^{-|q|}\right) .
$$


If $p_{1}(0) p_{2}(0) \geq 0$ it is known [CM2] that there is a unique solution to the Camassa-Holm equation corresponding to any choice of $q_{1}(0)$ and $q_{2}(0)$. On the other hand, within the setting $p_{1}(0) p_{2}(0)<0$ we encounter the peakon-antipeakon interaction, corresponding to the choice

$$
p_{1}(0)=-p_{2}(0), \quad q_{1}(0)=-q_{2}(0) .
$$

In view of (5.5) we have $P=Q=0$ so that

$$
p_{1}(t)=-p_{2}(t), \quad q_{1}(t)=-q_{2}(t), \quad p(t)=2 p_{1}(t), \quad q(t)=2 q_{1}(t), \quad t \geq 0 .
$$

Case I: $p(0) q(0)>0$

Notice that if $u$ is a solution to (1.1), so is $\tilde{u}(t, x)=-u(t,-x)$. Without loss of generality we may therefore assume that $p(0)>0$ and $q(0)>0$. By continuity, the inequalities $p(t)>0$ and $q(t)>0$ hold up to some maximal $T>0$. On $[0, T)$ we have

$$
\left\{\begin{array}{l}
p^{\prime}=-\frac{p^{2}}{2} e^{-q}, \\
q^{\prime}=p\left(1-e^{-q}\right),
\end{array}\right.
$$

whereas the Hamiltonian yields the invariant

$$
p^{2}\left(1-e^{-q}\right)=H_{0}^{2}>0, \quad t \in[0, T) .
$$

Therefore $p^{\prime}=\frac{H_{0}^{2}-p^{2}}{2}$ on $(0, T)$, so that

$$
p(t)=H_{0} \frac{\left[p(0)+H_{0}\right] e^{H_{0} t}+\left[p(0)-H_{0}\right]}{\left[p(0)+H_{0}\right] e^{H_{0} t}-\left[p(0)-H_{0}\right]}, \quad t \in[0, T) .
$$

On the other hand, since $q^{\prime}=\frac{H_{0}^{2}}{p}$ on $(0, T)$, we get

$$
q(t)=q(0)+2 \ln \frac{\left[p(0)+H_{0}\right] e^{H_{0} t / 2}+\left[p(0)-H_{0}\right] e^{-H_{0} t / 2}}{2 p(0)}, \quad t \in[0, T) .
$$

But (5.6) shows that $p(0)>H_{0}$, which by (5.7)-(5.8) yields $T=\infty$. Consequently, no wave breaking takes place in this case.

Case II: $p(0) q(0)<0$

In view of the symmetry property of the Camassa-Holm equation pointed out before, we may assume that $p(0)>0$ whereas $q(0)<0$. For some maximal time $T>0$ we will have $p(t)>0$ and $q(t)<0$ on $[0, T)$. On $[0, T)$ we then have

$$
\left\{\begin{array}{l}
p^{\prime}=\frac{p^{2}}{2} e^{q}, \\
q^{\prime}=p\left(1-e^{q}\right),
\end{array}\right.
$$


whereas the Hamiltonian yields the invariant

$$
p^{2}\left(1-e^{q}\right)=H_{0}^{2}>0, \quad t \in[0, T) .
$$

Therefore

$$
p^{\prime}=\frac{p^{2}-H_{0}^{2}}{2}
$$

on $(0, T)$, so that

$$
p(t)=H_{0} \frac{\left[p(0)+H_{0}\right]+\left[p(0)-H_{0}\right] e^{H_{0} t}}{\left[p(0)+H_{0}\right]-\left[p(0)-H_{0}\right] e^{H_{0} t}}, \quad t \in[0, T) .
$$

On the other hand, since $q^{\prime}=\frac{H_{0}^{2}}{p}$ on $(0, T)$, we get

$$
q(t)=q(0)-2 \ln \frac{\left[p(0)+H_{0}\right] e^{-H_{0} t / 2}+\left[p(0)-H_{0}\right] e^{H_{0} t / 2}}{2 p(0)}, \quad t \in[0, T) .
$$

Since $p(0)>H_{0}$ by (5.9), from (5.11)-(5.12) we infer that

$$
T=\frac{1}{H_{0}} \ln \frac{p(0)+H_{0}}{p(0)-H_{0}}
$$

and

$$
\lim _{t \uparrow T} p(t)=\infty
$$

Since $q(0)=\ln \frac{p^{2}(0)-H_{0}^{2}}{p^{2}(0)}$ by (5.10), from (5.12) we infer

$$
\lim _{t \uparrow T} q(t)=0 .
$$

From (5.14)-(5.15) we infer that at breaking time $T>0$ we have

$$
\lim _{t \uparrow T} u(t, x)=0 \quad \text { for every } \quad x \in \mathbb{R} .
$$

On the other hand, the explicit formulas

$u_{x}^{2}(t, x)=\left\{\begin{array}{l}\frac{p^{2}}{4}\left(e^{q}-1\right)\left(1-e^{-q}\right) e^{2 x} \quad \text { for } \quad x \leq q_{1}(t) \\ p^{2} e^{q} \cosh ^{2}(x) \text { for } \quad x \in\left(q_{1}(t), q_{2}(t)\right) \\ \frac{p^{2}}{4}\left(e^{q}-1\right)\left(1-e^{-q}\right) e^{-2 x} \quad \text { for } \quad x \geq q_{2}(t)\end{array}=\left\{\begin{array}{l}\frac{H_{0}^{2}}{4}\left(e^{-q}-1\right) e^{2 x} \quad \text { for } \quad x \leq q_{1}(t) \\ \frac{e^{q} H_{0}^{2}}{1-e^{q}} \cosh ^{2}(x) \text { for } \quad x \in\left(q_{1}(t), q_{2}(t)\right) \\ \frac{H_{0}^{2}}{4}\left(e^{-q}-1\right) e^{-2 x} \text { for } x \geq q_{2}(t)\end{array}\right.\right.$

valid for $t \in[0, T)$, show that the measure with density $u_{x}^{2}(t, \cdot)$ approaches weakly a Dirac mass concentrated at the origin, as $t \uparrow T$. Furthermore, a straightforward calculation shows that the total amount of energy concentrated in the interval between the two extremes (crest and trough) of the wave equals

$$
\int_{q_{1}(t)}^{q_{2}(t)}\left(u^{2}(t, x)+u_{x}^{2}(t, x)\right) d x=\frac{p^{2}(t)}{2}\left(1-e^{2 q(t)}\right), \quad t \in[0, T) .
$$


On the other hand, the total energy equals

$$
\|u(t, \cdot)\|_{H^{1}}^{2}=p^{2}(t)\left(1-e^{q(t)}\right)=H_{0}^{2} .
$$

In view of $(5.15)$, we have that

$$
\lim _{t \uparrow T} \int_{q_{1}(t)}^{q_{2}(t)}\left(u^{2}(t, x)+u_{x}^{2}(t, x)\right) d x=H_{0}^{2}
$$

so that, as $t$ approaches $T$, an increasingly large portion of the energy is concentrated within the interval $\left[q_{1}(t), q_{2}(t)\right]$. In the limit $t \uparrow T$ all the energy becomes concentrated at the single point $x=0$.

To resolve the singularity arising at $t=T$ we introduce the variable

$$
w(t)=2 \arctan \frac{p(t)}{H_{0}}, \quad t \geq 0 .
$$

Then (5.10) yields

$$
w^{\prime}(t)=-H_{0}\left(\frac{1-\left(\frac{p(t)}{H_{0}}\right)^{2}}{1+\left(\frac{p(t)}{H_{0}}\right)^{2}}\right)=-H_{0}\left(\frac{1-\tan ^{2} \frac{w(t)}{2}}{1+\tan ^{2} \frac{w(t)}{2}}\right)=-H_{0} \cos w(t) .
$$

Written in this new variable, the solution is smooth. Actually, it can be continued analytically for all $t \in \mathbb{R}$. In this case, for $t>T$, the solution would contain two peakons moving away from each other, i.e., with $\left(p_{1}-p_{2}\right)\left(q_{1}-q_{2}\right)>0$, as in Case I.

Notice that this solution $u=u(t, x)$ in (5.1) preserves the total energy at all times except at $t=T$, when $u(T, x) \equiv 0$.

By direct inspection, one can check that this conservative multipeakon solution coincides with the solution constructed through our variable transformations in Sections 2-4. Clearly, these solutions by themselves do not satisfy the semigroup property. For $t>T, u(t, \cdot) \neq 0$, while the solution to the Cauchy problem with initial data $u(T, x) \equiv 0$, as in (5.16), should vanish identically.

To obtain a semigroup, it is clear that we need to retain some additional information about the solutions. In particular, we need to distinguish between an initial data which is identically zero, and an initial condition $\bar{u}$ with $\bar{u}(x) \equiv 0$ but where $u_{x}^{2}$ formally consists of a unit Dirac mass at the origin, a situation encountered in the case of the peakon-antipeakon interaction at the breaking time $t=T$. For this purpose, we consider the domain $\mathcal{D}$ consisting of all couples $(u, \mu)$, where $u \in H^{1}(\mathbb{R})$, while $\mu$ is a positive Radon measure on $\mathbb{R}$ satisfying

$$
d \mu^{a}=u_{x}^{2} d x
$$

In other words, splitting $\mu=\mu^{a}+\mu^{s}$ as the sum of an absolutely continuous and a singular part, we require that the absolutely continuous part have density $u_{x}^{2}$ w.r.t. Lebesgue measure. We call $\mathcal{M}(\mathbb{R})$ the metric space of all bounded Radon measures on $\mathbb{R}$, endowed with the topology of weak convergence. 
Given $(\bar{u}, \bar{\mu}) \in \mathcal{D}$, we define the map $\xi \mapsto \bar{y}(\xi)$ by setting

$$
\begin{array}{ll}
\bar{y}(\xi) \doteq \sup \{x ; \quad x+\bar{\mu}([0, x]) \leq \xi\} & \text { if } \xi \geq 0, \\
\bar{y}(\xi) \doteq \inf \{x ;|x|+\bar{\mu}([x, 0[) \leq|\xi|\} & \text { if } \xi<0 .
\end{array}
$$

This definition is designed so that, for any Borel set $J \subset \mathbb{R}$ we have

$$
\bar{\mu}(J)+\operatorname{meas}(J)=\operatorname{meas}\{\xi \in \mathbb{R} ; \quad \bar{y}(\xi) \in J\} .
$$

Notice that this reduces to (2.1) in the case where $\bar{\mu}$ is absolutely continuous. In all cases, the map $\xi \mapsto \bar{y}(\xi)$ is Lipschitz continuous with constant 1 , hence it is a.e. differentiable. We now solve the system of equations (3.1) with initial data

$$
\begin{gathered}
\bar{u}(\xi)=\bar{u}(\bar{y}(\xi)), \quad q(\xi) \equiv 1, \\
\begin{cases}\bar{v}(\xi)=2 \arctan u_{x}(\bar{y}(\xi))=2 \arctan \bar{u}_{\xi}(\xi) \cdot \frac{d \xi}{d \bar{y}} & \text { if } d \bar{y} / d \xi>0, \\
\bar{v}(\xi)=\pi & \text { if } d \bar{y} / d \xi=0 .\end{cases}
\end{gathered}
$$

In turn, from this solution $(u, v, q)$ we recover a mapping $t \mapsto\left(u(t), \mu_{(t)}\right) \in H^{1} \times \mathcal{M}$ defined by (4.1), (4.3) together with

$$
\mu_{(t)}([a, b])=\int_{\{\xi ; y(t, \xi) \in[a, b]\}} \sin ^{2} \frac{v(t, \xi)}{2} q(t, \xi) d \xi
$$

Our main result in this section is the following.

Theorem 3. There exists a continuous semigroup $\Psi: \mathcal{D} \times[0, \infty) \mapsto \mathcal{D}$ whose trajectories $t \mapsto$ $\left(u(t), \mu_{(t)}\right)=\Psi_{t}(\bar{u}, \bar{\mu})$ have the following properties:

(i) The function $u$ provides a solution to the Cauchy problem (1.1)-(1.2) in the sense of Definition 1 , while the family of measures $\left\{\mu_{(t)}, t \in \mathbb{R}\right\}$ provides a measure valued solution $w$ to the linear transport equation with source

$$
w_{t}+(u w)_{x}=2\left(u^{2}-P\right) u_{x}
$$

(ii) For a.e. $t \in \mathbb{R}$ the measure $\mu_{(t)}$ is absolutely continuous. Its density w.r.t. Lebesgue measure is given by

$$
d \mu_{(t)}=u_{x}^{2}(t, \cdot) d x .
$$

(iii) If $\bar{u}_{n} \rightarrow \bar{u}$ in $H^{1}(\mathbb{R})$ and $\bar{\mu}_{n} \rightarrow \bar{u}$ weakly, then $u_{n}(t, x) \rightarrow u(t, x)$ uniformly for $(t, x)$ in bounded sets.

Proof. Most of the above statements already follow from the analysis in the previous sections. Indeed, we have already proved that the function $u=u(t, x)$ defined at $(4.1),(4.3)$ provides a solution to the Camassa-Holm equation (1.1)-(1.2). 
As in (3.31), call $\mathcal{N} \subset \mathbb{R}$ the set of times where meas $\{\xi ; \cos v(t, \xi)=-1\}>0$ For $t \notin$ $\mathcal{N}$, the measure $\mu_{(t)}$ is precisely the absolutely continuous Radon measure having density $u_{x}^{2}$ w.r.t. Lebesgue measure. On the other hand, our construction implies that, for $t \in \mathcal{N}$, the measure $\mu_{(t)}$ is the weak limit of the measures $\mu_{(s)}$, as $s \rightarrow t, s \notin \mathcal{N}$. Since the set $\mathcal{N}$ has measure zero, in view of (1.6) we deduce (5.20). Notice that this equation can be formulated more precisely as

$$
\left.\int_{\mathbb{R}} \varphi_{t} d \mu_{(t)}\right|_{t_{1}} ^{t_{2}}=\int_{t_{1}}^{t_{2}} \int_{\mathbb{R}} \varphi_{x} u d \mu_{(t)} d t-2 \int_{t_{1}}^{t_{2}} \int_{\mathbb{R}}\left(u^{2}-P\right) u_{x} \varphi d x d t
$$

for every $t_{2}>t_{1} \geq 0$ and any function $\varphi \in C^{1}\left(\mathbb{R}_{+} \times \mathbb{R}\right)$ with compact support in $(0, \infty) \times \mathbb{R}$.

If $\left(\bar{u}_{n}, \bar{\mu}_{n}\right)$ is a sequence of initial data with $\bar{u}_{n} \rightarrow \bar{u}$ in $H^{1}(\mathbb{R})$ and $\bar{\mu}_{n} \rightarrow \bar{\mu}$ weakly, then at time $t=0$ this implies

$$
\sup _{\xi \in \mathbb{R}}\left|y_{n}(0, \xi)-y(0, \xi)\right| \rightarrow 0, \quad \sup _{\xi \in \mathbb{R}}\left|u_{n}(0, \xi)-u(0, \xi)\right| \rightarrow 0
$$

Moreover,

$$
\left\|v_{n}(0, \cdot)-v(0, \cdot)\right\|_{\mathbf{L}^{2}} \rightarrow 0 .
$$

By the continuity of the solution of (3.1) on the initial data, this implies $u_{n}(t, \xi) \rightarrow u(t, \xi)$, uniformly for $t, \xi$ in bounded sets. Returning to the original coordinates, this yields the convergence

$$
y_{n}(t, \xi) \rightarrow y(t, \xi), \quad u(t, x) \rightarrow u_{n}(t, x),
$$

uniformly on bounded sets.

To complete the proof of Theorem 3 it now remains to prove the semigroup property:

$$
\Psi_{t} \circ \Psi_{s}(\bar{u}, \bar{\mu})=\Psi_{t+s}(\bar{u}, \bar{\mu}) .
$$

Starting with $(\bar{u}, \bar{\mu}) \in \mathcal{D}$, let $U(\tau, \xi)=(u(\tau, \xi), v(\tau, \xi), q(\tau, \xi))$ be the unique solution of (3.1) with initial data $(u(0, \xi), v(0, \xi), 1)$, defined for all $\tau \geq 0$. Then $\Psi_{t+s}(\bar{u}, \bar{\mu})$ is obtained from $U(t+s, \xi)$ via (4.1), (4.3) and (5.19). To obtain $\Psi_{t} \circ \Psi_{s}(\bar{u}, \bar{\mu})$, one proceeds as follows. If $\tilde{U}(\tau, \xi), \tau \geq 0$, is the solution of $(3.1)$ with initial data $(u(s, \xi), v(s, \xi), 1)$, then $\Psi_{t} \circ \Psi_{s}(\bar{u}, \bar{\mu})$ is obtained from $\tilde{U}(t, \xi)$ by means of (4.1), (4.3) and (5.19). Notice that $U(t+\tau, \xi)$ with $\tau \geq 0$ is the solution of (3.1) with initial data $(u(s, \xi), v(s, \xi), q(s, \xi))$. We claim that

$$
U(t+s, \xi)=\tilde{U}(t, \tilde{\xi})
$$

where $\tilde{\xi}$ is a bi-Lipschitz parametrization of the $\xi$-variable with

$$
\frac{d \tilde{\xi}}{d \xi}=\frac{q(\tau, \xi)}{\tilde{q}(\tau, \xi)} \quad \text { at time } \quad \tau \geq 0
$$

Indeed, (2.8)-(2.9) and the form of (3.1) confirm the validity of (5.23) in view of the change of variables (5.24). The fact that $\xi \mapsto \tilde{\xi}$ is a bi-Lipschitz parametrization follows at once if we notice that the linearity of the third equation in (3.1) with respect to $q$ yields

$$
\partial_{\tau} \frac{q(\tau, \xi)}{\tilde{q}(\tau, \xi)}=\left(\left(u^{2}+\frac{1}{2}-P\right) \sin v-\left(\tilde{u}^{2}+\frac{1}{2}-\tilde{P}\right) \sin \tilde{v}\right) \frac{q(\tau, \xi)}{\tilde{q}(\tau, \xi)}
$$

and the factor of $\frac{q(\tau, \xi)}{\tilde{q}(\tau, \xi)}$ on the right-hand side is uniformly bounded. We thus established the validity of (5.23). A glance at (4.1), (4.2) and (5.19) confirms now that (5.22) holds. 


\section{References}

[A] N. Aronszajn, Differentiability of Lipschitzian mappings between Banach spaces, Studia Math. 57 (1976), 147-190.

[BSS1] R. Beals, D. Sattinger and J. Szmigielski, Multi-peakons and a theorem of Stieltjes, Inverse Problems 15 (1999), L1-L4.

[BSS2] R. Beals, D. Sattinger and J. Szmigielski, Multipeakons and the classical moment problem, Adv. Math. 154 (2000), 229-257.

[BSS3] R. Beals, D. Sattinger and J. Szmigielski, Peakon-antipeakon interaction, J. Nonlinear Math. Phys. 8 (2001), 23-27.

[BZZ] A. Bressan, P. Zhang, and Y. Zheng, On asymptotic variational wave equations, Arch. Rat. Mech. Anal., to appear.

[CH] R. Camassa and D. D. Holm, An integrable shallow water equation with peaked solitons, Phys. Rev. Lett. 71 (1993), 1661-1664.

[C1] A. Constantin, Existence of permanent and breaking waves for a shallow water equation: a geometric approach, Ann. Inst. Fourier (Grenoble) 50 (2000), 321-362.

[C2] A. Constantin, On the scattering problem for the Camassa-Holm equation, Proc. Roy. Soc. London Ser. A 457 (2001), 953-970.

[CE1] A. Constantin and J. Escher, Global existence and blow-up for a shallow water equation, Ann. Scuola Norm. Sup. Pisa 26 (1998), 303-328.

[CE2] A. Constantin and J. Escher, Wave breaking for nonlinear nonlocal shallow water equations, Acta Mathematica 181 (1998), 229-243.

[CM1] A. Constantin and H. P. McKean, A shallow water equation on the circle, Comm. Pure Appl. Math. 52 (1999), 949-982.

[CM2] A. Constantin and L. Molinet, Global weak solutions for a shallow water equation, Comm. Math. Phys. 211 (2000), 45-61.

[CS] A. Constantin and W. Strauss, Stability of peakons, Comm. Pure Appl. Math. 53 (2000), 603-610.

[D] J. Diéudonne, Foundations of Modern Analysis. Academic Press, New York, 1960.

[EG] L. C. Evans and R. F. Gariepy, Measure Theory and Fine Properties of Functions, Studies in Advanced Mathematics, CRC Press, Boca Raton, FL, 1992.

[FF] A. Fokas and B. Fuchssteiner, Symplectic structures, their Bäcklund transformation and hereditary symmetries, Physica D 4 (1981), 47-66.

[L] J. Lenells, Conservation laws of the Camassa-Holm equation, J. Phys. A 38 (2005), 869-880. 
[J] R. S. Johnson, Camassa-Holm, Korteweg-de Vries and related models for water waves, J. Fluid Mech. 455 (2002), 63-82.

[M] H. P. McKean, Fredholm determinants and the Camassa-Holm hierarchy, Comm. Pure Appl. Math. 56 (2003), 638-680.

[W] E. Wahlen, On the peakon-antipeakon interaction, Dynamics Cont. Discr. Impuls. Systems, to appear.

[XZ1 Z. Xin and P. Zhang, On the weak solutions to a shallow water equation, Comm. Pure Appl. Math. 53 (2000), 1411-1433.

[XZ2] Z. Xin and P. Zhang, On the uniqueness and large time behavior of the weak solutions to a shallow water equation, Comm. Partial Differential Equations 27 (2002), 1815-1844. 